\title{
Anti-thyroid drugs as treatment for neutropenia in Graves' thyrotoxicosis
}

\author{
N Aggarwal" ${ }^{\uparrow}$, Su-ann Tee*, W Saqib*, T Fretwell*, G Summerfield ${ }^{\S}$,S Razvi*\# \\ " Department of Diabetes \& Endocrinology, The James Cook University Hospital, Middlesbrough,UK \\ *Department of Diabetes \& Endocrinology, Queen Elizabeth Hospital, Gateshead, UK \\ $\S$ Department of Haematology, Queen Elizabeth Hospital, Gateshead, UK \\ \# Institute of Human Genetics, Newcastle University, Newcastle upon Tyne, UK
}

Background: Neutropenia due to anti-thyroid drugs (ATD) in Graves' disease is rare but well recognised. However, the effect of hyperthyroidism, and of ATD, on absolute and relative neutrophil counts in patients with Graves' disease is unclear.

Methods: Neutrophil counts were noted in consecutive patients with newly diagnosed Graves' thyrotoxicosis in 2010-13 ( $n=147)$. Neutrophil levels were further noted once patients had been treated with ATD for at least 3 months and were rendered euthyroid. A neutrophil count of $<2 \times 10^{9} / \mathrm{L}$ was classed as neutropenia. Neutrophil levels at baseline and at euthyroid state were compared by student's paired $t$-test. Multivariable linear regression analysis was performed to ascertain factors affecting change in neutrophil levels. All the results were also analysed according to smoking status.

Results: After multivariable regression analysis, severity of hyperthyroidism $(p<0.005)$ and non smoking status $(p=0.02)$ were the only independent predictors of neutropenia and there was no association with TBII levels.

\section{The incidence of neutropenia in non-smokers} (20.2\%) was almost double of that in current smokers (11.1\%).

All the patients who were initially neutropenic had normal neutrophil counts after euthyroidism was achieved following ATD therapy. Improvement in neutrophil count was related to reduction in thyroid hormone levels $(\mathrm{P}<0.001)$ and was more pronounced in males.

Conclusion: Neutropenia is frequent $(16.3 \%)$ in untreated Graves' thyrotoxicosis. Neutropenia is related to the degree of severity of hyperthyroidism, is more common in non-smokers and unrelated to thyroid autoimmunity. We suggest that neutrophil levels should be checked in newly diagnosed patients with Graves' thyrotoxicosis prior to commencing ATD therapy as otherwise low levels may incorrectly be attributed to ATD therapy. In addition, the mechanism underlying the effect of thyroid hormones on neutrophil levels needs to be studied.

\begin{tabular}{|c|c|c|c|}
\hline \multicolumn{4}{|c|}{ Neutropenic and Non-neutropenic groups } \\
\hline $\begin{array}{c}\text { Mean }( \pm S D) \\
\text { or } \\
n(\%)\end{array}$ & $\begin{array}{l}\text { Neutropenic } \\
n=24(16.3 \%)\end{array}$ & $\begin{array}{c}\text { Non-neutropenic } \\
n=123(83.7 \%)\end{array}$ & P value \\
\hline Age & $42.1 \pm 13.2$ & $50.3 \pm 15.5$ & 0.02 \\
\hline Male sex & $4(16.7 \%)$ & $19(15.4 \%)$ & NS \\
\hline $\begin{array}{l}\text { Current } \\
\text { smokers }\end{array}$ & $5(20.8 \%)$ & $42(34.1 \%)$ & $<0.01$ \\
\hline Orbitopathy & $6(25 \%)$ & $19(15.4 \%)$ & 0.01 \\
\hline FT4 (pmol/L) & $67.7 \pm 27.9$ & $45.3 \pm 22.9$ & $<0.001$ \\
\hline FT3 (pmol/L) & $28.7 \pm 12.2$ & $18.7 \pm 11.2$ & $<0.001$ \\
\hline TBII & $15.1 \pm 12.4$ & $11.6 \pm 9.8$ & NS (Log) \\
\hline
\end{tabular}

Smokers and non smokers

\begin{tabular}{|c|c|c|c|}
\hline n (\%) & $\begin{array}{c}\text { Current smokers } \\
\mathbf{n = 4 5}\end{array}$ & $\begin{array}{c}\text { Current non-smokers } \\
\mathbf{n = 9 4}\end{array}$ & P value \\
\hline Neutropenia & $5(11.1 \%)$ & $19(20.2 \%)$ & $<0.001$ \\
\hline
\end{tabular}

Results after euthyroidism

\begin{tabular}{|c|c|c|c|c|c|c|}
\hline \multicolumn{7}{|c|}{ Neutropenic and Non-neutropenic groups } \\
\hline & \multicolumn{3}{|c|}{$\begin{array}{l}\text { Neutropaenic group } \\
\qquad(n=21)\end{array}$} & \multicolumn{3}{|c|}{$\begin{array}{l}\text { Non neutropaenic } \\
\text { group }(n=74)\end{array}$} \\
\hline & Baseline & $\begin{array}{c}\text { After } \\
\text { euthyroidism }\end{array}$ & $P$ value & Baseline & $\begin{array}{c}\text { After } \\
\text { euthyroidism }\end{array}$ & P va \\
\hline WBC & $3.9 \pm 1.2$ & $5.6 \pm 1.5$ & .001 & $6.9 \pm 1.7$ & $7.6 \pm 2$ & 0.005 \\
\hline Neutrophils & $\begin{array}{l}1.6 \pm 0.3 \\
(39.7 \%)\end{array}$ & $\begin{array}{l}3.4 \pm 1.2 \\
(59.9 \%)\end{array}$ & $<0.001$ & $\begin{array}{l}3.9 \pm 1.3 \\
(56.3 \%)\end{array}$ & $\begin{array}{l}4.8 \pm 1.7 \\
(63.4 \%)\end{array}$ & $<0$ \\
\hline ymphocytes & $\begin{array}{l}1.7 \pm 0.9 \\
(43.3 \%)\end{array}$ & $\begin{array}{l}1.6 \pm 0.8 \\
(29.3 \%)\end{array}$ & NS & $\begin{array}{l}2.2 \pm 0.6 \\
(30.7 \%)\end{array}$ & $\begin{array}{c}2.0 \pm 0.6 \\
(25.4 \%)\end{array}$ & 0.02 \\
\hline Taemoglobin & $12.4 \pm 1.1$ & $13.5 \pm 1.3$ & $<0.001$ & $13.2 \pm 1.3$ & $13.7 \pm 1.4$ & 0.03 \\
\hline Platelets & $220 \pm 59.1$ & $247 \pm 67.8$ & $<0.001$ & $248 \pm 52$ & $257 \pm 60$ & NS \\
\hline
\end{tabular}

Smokers and non smokers

\begin{tabular}{|c|c|c|c|c|c|c|}
\hline & \multicolumn{2}{|c|}{$\begin{array}{c}\text { Current smokers } \\
(\mathbf{n = 3 1 )}\end{array}$} & \multicolumn{3}{c|}{$\begin{array}{c}\text { Current non-smokers } \\
(\mathbf{n = 6 1 )}\end{array}$} \\
\hline & Baseline & $\begin{array}{c}\text { After } \\
\text { euthyroidism }\end{array}$ & P value & Baseline & $\begin{array}{c}\text { After } \\
\text { euthyroidism }\end{array}$ & P value \\
\hline WBC & $7.4 \pm 2$ & $8.0 \pm 2.1$ & 0.3 & $5.7 \pm 1.8$ & $6.8 \pm 1.9$ & $<0.001$ \\
\hline \multirow{2}{*}{ Neutrophils } & $4.1 \pm 1.6$ & $4.9 \pm 1.7$ & & $3.0 \pm 1.2$ & $4.3 \pm 1.7$ & $<0.001$ \\
& $(55.4 \%)$ & $(61.1 \%)$ & 0.07 & $(52.8 \%)$ & $(63.7 \%)$ & \\
\hline Lymphocytes & $2.4 \pm 0.8$ & $2.2 \pm 0.7$ & & $1.9 \pm 0.6$ & $1.8 \pm 0.6$ & 0.1 \\
& $(32.2 \%)$ & $(28.1 \%)$ & 0.5 & $(32.7 \%)$ & $(24.9 \%)$ & 0.1 \\
\hline
\end{tabular}

